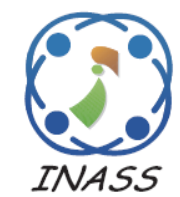

http://www.inass.org/

\title{
Energy Efficient Evolved Node Base Station and Relay Station Placement Scheme for Green Cellular Networks
}

\author{
Arthi Murugadass $^{1} \quad$ Vinoth Babu Kumaravelu $^{2 *} \quad$ SunilKumar Reddy Thippayagari $^{1}$ \\ ${ }^{I}$ Department of Computer Science and Engineering, \\ Sri Venkateswara College of Engineering \& Technology, Chittoor, Andhra Pradesh, India \\ ${ }^{2}$ Department of Communication Engineering, School of Electronics Engineering, \\ Vellore Institute of Technology, Vellore, Tamil Nadu, India \\ * Corresponding author's Email: vinothbab@gmail.com
}

\begin{abstract}
A novel placement of evolved node base station (eNB) and relay station (RS) is a standout amongst the most vital key necessities of multi-hop relay (MHR) networks to accomplish higher transmission rates and coverage ratio. Inappropriate eNB and RS placement leads to loss in network throughput and energy efficiency. This work focuses on quality of service (QoS) improvement based on effective eNB and RS placement for green cellular networks. An efficient modified uniform clustering based placement scheme is proposed in this work, which selects appropriate placement locations for eNB and RS from the candidate locations. The proposed scheme combines uniform clustering based placement scheme, load aware spectral efficient routing (LASER) scheme and throughput oriented (TO) selection rule to offer higher throughput and power consumption effectiveness performances even under network imbalance conditions. The simulation results accept the superior performance of our proposed scheme over the other conventional schemes.
\end{abstract}

Keywords: Coverage ratio, eNB and RS placement, Network imbalance, Path determination, Placement budget, Throughput.

\section{Introduction}

The growth in the usage of smart devices and energy hungry applications (online gaming, social networks, high definition video streaming etc.) increases the energy consumption [1]. CISCO's global visual networking index (VNI) predicted that there will be an 8-fold growth in global mobile traffic from 2015 to 2020 [2]. They have also predicted that the number of connected devices will be around 11.6 billion by 2020 , which will be larger than that time world's population. In order to meet such a huge traffic demand, dense deployment of Macro eNBs are required. Due to higher energy consumption, the conventional eNBs are turned out to be inefficient. The increase in the Macro eNBs increases the energy consumption, which in turn increases the operational cost for the network operators $[3,4]$.
Various solutions have been proposed in the literature to limit energy emission and to improve the coverage and capacity. Most of the existing solutions focus on limiting energy consumption at the user side to maximize life time of the mobile batteries. Presently researchers and operators are developing intelligent algorithms to improve the energy efficiency at the serving node side.

The report released by CISCO and Huawei states that $70 \%$ of the data traffic is caused by indoor users [5]. It is very important for mobile network operators (MNO) to improve the data rate and coverage of indoor users. With a specific end goal to accomplish perpetually expanding interest on information rate and QoS, the third generation partnership project (3GPP) have presented a standard called long term evolution-advanced (LTEA), which considers new transmission schemes like carrier aggregation, MHR and coordinated 
multipoint transmission and reception (CoMP) [6]. For a long-time scale energy management, macro cells are replaced with small cells in high traffic regions. Ultra-dense networks (UDN) based on small cells are the promising solution to improve the energy efficiency of wireless networks [7, 8]. This solution will also satisfy the customers in terms of coverage and capacity while handling drastic mobile data traffic. Since the placement and maintenance cost of the RS is much lower than the eNB, RS based small cell systems are effectively considered in the standardization process of next generation mobile broadband standards like IEEE 802.16j, IEEE $802.16 \mathrm{~m}$ and LTE-A. RS placement is extremely useful in the areas where wired backhaul link is expensive and unavailable [9].

Identifying the placement locations of eNB and RS have become a severe issue in MHR network. Selecting improper placement locations lead to loss in system throughput and energy efficiency [10]. Increase in the number of indirect transmissions may prompt the transmission delay and loss in system throughput [11-13]. Improper placements will also lead to path selection issue, which reduces the system quality if left uncared $[14,15]$. 3GPP LTE-A, IEEE 802.16j and IEEE 802.16m standards failed to give suitable mechanism for eNB and RS placement. Since it is an open issue, many of the working groups have proposed various placement schemes.

In many of the works, the placement mechanism for eNB is not examined. Only a few works discusses the neediness of eNB and RS placement together $[13,14,16]$. H. C. Lu and W. Liao [13] proposed a joint BS and RS placement (JBRP) scheme for IEEE $802.16 \mathrm{j}$ networks. The placement problem is illustrated with an integer linear programming model. The proposed scheme maximizes the network throughput by maintaining the total placement cost within the limits of the maximum allowed placement budget. JBRP scheme addresses both the coverage and budget constraints. But this scheme experiences severe quality loss from network imbalance issues. The imbalance in traffic leads to a larger packet queuing delay and loss in system throughput. This is a severe issue in terms of both customer and operator point of view. The unsatisfied customer will switch their service to other competing operator. The authors in [13] also assumed that the candidate locations of RSs are same as the locations of user equipment (UE) and the candidate locations of eNBs are the corners of demand nodes (DN). These assumptions are unreasonable and unrealizable.
Y. Chang and Y. S. Lin [10] proposed a novel uniform clustering eNB and RS placement scheme based on modified k-means algorithm. The same authors have also proposed an eNB and RS placement scheme based on fuzzy inference mechanism [17]. The fuzzy inference engine uses traffic ratio and coverage ratio as the two input fuzzy parameters. Both these algorithms, work in two phases, namely eNB-RS selection phase and eNB-RS placement phase. Despite the fact that these schemes offer better throughput and coverage over the JBRP scheme, they experience various issues. Some of them are listed here: The authors have proposed two different algorithms satisfying coverage and budget constraints separately. Based on the demand, eNB has to select one among these two algorithms. The robustness of the eNB in enabling proper switching between these two algorithms is not discussed in the current work. The capacity of the RS is limited compared to the eNB. The network imbalance issue rises whenever there is a non-uniform traffic demand from RSs [14]. This leads to a disappointing network throughput. The impact of network imbalance issue on eNB and RS placement is not examined in the present work. Inter $\mathrm{RS}$ interference is the other severe issue in MHR network, which is also not addressed in the current work. The simulation results show that the average network throughput obtained is less than the conventional throughput based placement schemes [10]. There are no special considerations in the present work for enhancing the system throughput. An adaptive cost based RS deployment (ACRD) scheme is proposed in $[18,19]$ by considering five impact factors like transmission quality, placement cost, coverage ratio with and without population and overlapping index. This scheme aims to deploy more number of RSs on the highly populated areas. But this scheme is tested for a smaller $2 \mathrm{~km} \mathrm{X} 2 \mathrm{~km}$ area. The increase in the RS deployment combinations also increases the computational complexity. A new eNB and RS placement scheme should address few or all of the issues mentioned above.

Our proposed eNB and RS placement scheme combines best components of different algorithms to address some of the above mentioned issues. The two phase transmission nature of MHR systems debases the system capacity furthermore builds the transmission delay $[11,12]$. Our proposed scheme selects for indirect transmission only when it is needed. It embraces the idea of TO selection rule, which was initially proposed by J. H. Huang [12]. It has been proved that TO based selection rule, offers 
better performance over the signal strength oriented selection rule.

Due to the introduction of RSs, each UE may receive signals from eNB in various paths. Path determination is one of the critical issues in MHR networks [14]. Improper path determination may prompt loss in system throughput and energy efficiency and increase in transmission delay [4]. A large number of the path determination schemes proposed in the literature have not considered the network imbalance issue. S. S. Wang [14] proposed a LASER scheme, which uses link spectral efficiency as the path determination metric and proved to offer better performance even under network imbalance conditions. Hence, our proposed scheme additionally adopts LASER based path determination to address the network imbalance issue.

Placing a RS in a properly covered region is meaningless. It doesn't help in enhancing the system throughput rather it decreases the system throughput because of the inter RS interference. In this manner, the proposed work supports setting the RSs on the cell edges with adequately far separation in order to stay away from inter RS interference. To meet the expected system throughput and coverage while maintaining the placement cost low, the proposed scheme energizes setting more number of RSs than the eNBs. By including all the aforementioned factors, a new algorithm has been proposed in this work, which offers preferred QoS over the JBRP and uniform clustering based schemes. Our proposed modified uniform clustering based eNB and RS placement scheme has the adequacy to exhibit the network operator a quality and energy aware competent placement scheme for MHR networks.

The rest of the manuscript is organized in the following way. Section 2 presents the MHR system model and section 3 introduces the proposed eNB and RS placement scheme. Section 4 examines the simulation results and section 5 concludes the manuscript by highlighting the future works.

\section{System model}

We consider a geographic region where UE are arbitrarily placed with non-uniform traffic demands. eNB has the direct link to backhaul networks. eNBs are in charge of data transmission between UE and eNB.It also acts as the deciding authority in path determination when there exists multiple paths between eNB and UE. All the eNBs are associated with mobile switching centre (MSC), which acts as a gateway between the wired and wireless networks.

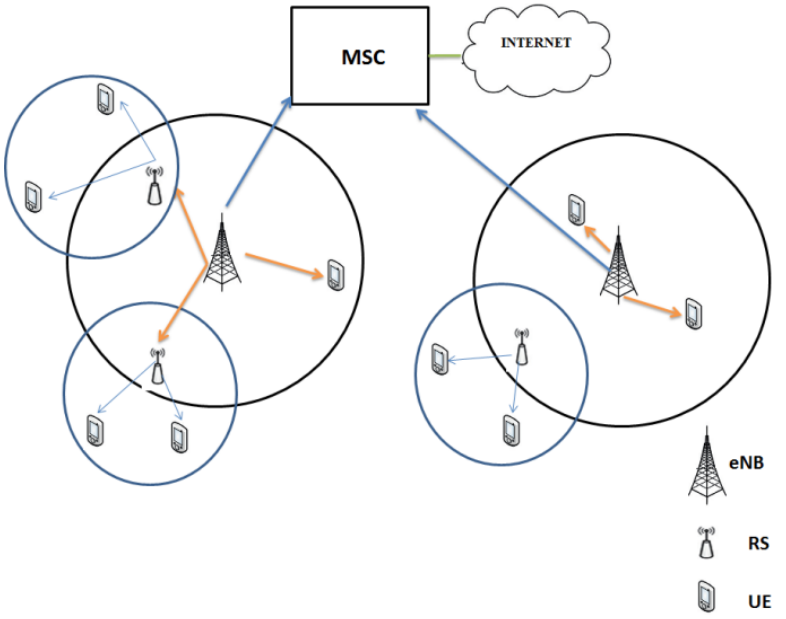

Figure. 1 MHR network architecture

The placement of eNBs and RSs ought to be efficient in a manner that every UE inside the geographic region must have a connection at least with an eNB or RS. The MHR network architecture model is illustrated in Fig.1.

Since we focus on coverage extension, the placement of Type I RSs is alone considered in this work. In any case, the proposed scheme can be extended to Type II RSs. A simple free space propagation model is assumed for path loss considerations. The signal to noise ratio (SNR) is given by [10],

$\operatorname{SNR}(d B)=10 \log _{10}\left(\frac{P_{t}}{\sigma_{n}^{2}}\right)\left(\frac{c}{4 \pi f_{c} d}\right)^{2}$

where $P_{t}$ is the transmitted signal power, $\sigma_{n}^{2}$ is the noise variance, $c$ is the velocity of light, $f_{c}$ is the carrier frequency and $d$ is the distance between the communicating nodes.

To build the QoS, the proposed scheme utilizes adaptive modulation and coding (AMC). eNB offers suitable modulation and coding scheme for every UE based on their channel conditions $[10,14]$. The AMC schemes utilized as a part of this work are listed in Table 1 [10, 17]. LTE-A and IEEE 802.16j standards support modulation schemes like binary phase shift keying (BPSK), quadrature phase shift keying (QPSK) and quadrature amplitude modulation (QAM). From Table 1, it is clear that the UEs with lower SNR are supported with lower burst profiles and UEs with high SNR is supported with higher burst profiles. The primary point of placing the RSs is to decrease the separation between the communication nodes and to improve the SNR so that the UEs achieve higher throughput constantly. 
Table 1. AMC scheme for link adaptation [10, 17]

\begin{tabular}{|c|c|c|c|c|c|}
\hline Burst Profile & Coding Rate & Modulation & $\begin{array}{c}\text { Distance between the } \\
\text { nodes (km) }\end{array}$ & SNR (dB) & $\begin{array}{c}\text { Data Rate } \\
\text { (Mbps) }\end{array}$ \\
\hline 1 & $1 / 2$ & BPSK & 3.20 & 3.00 & 1.269 \\
\hline 2 & $1 / 2$ & QPSK & 2.70 & 6.00 & 2.538 \\
\hline 3 & $3 / 4$ & QPSK & 2.50 & 8.50 & 3.816 \\
\hline 4 & $1 / 2$ & 16-QAM & 1.90 & 11.5 & 5.085 \\
\hline 5 & $3 / 4$ & 16-QAM & 1.70 & 15.0 & 7.623 \\
\hline 6 & $2 / 3$ & 64-QAM & 1.30 & 19.0 & 10.161 \\
\hline 7 & $3 / 4$ & 64-QAM & 1.20 & 21.0 & 11.439 \\
\hline
\end{tabular}

The eNB and RS placement problem is formulated as follows:

Define the eNB and RS candidate location vectors.

$\boldsymbol{B}^{c}=[1,2 \ldots b]$
$\boldsymbol{R}^{c}=[1,2 \ldots r]$

where $b$ and $r$ represents the number of candidate locations of eNB and RS respectively. [13]

The objective of the proposed algorithm is to

$\operatorname{Max}\left[\left(\sum_{u=1}^{T} R_{u}^{U E} \cdot D_{u}^{U E} \cdot \alpha_{u}\right)\left(\sum_{u=1}^{T} \alpha_{u}\right)\right]$

where $R_{u}^{U E}$ is the data transmission rate between MSC and $u^{\text {th }}$ UE, $D_{u}^{U E}$ is the traffic demand of $u^{\text {th }}$ $\mathrm{UE}$ and $\alpha_{u}$ is the binary function which is given by,

$\alpha_{u}= \begin{cases}1 & \text {;if } u^{\text {th }} U E \text { is connected with any } e N B \text { or } R S \\ 0 & ; \text {;else }\end{cases}$

Subjected to:

1. The inter RS interference constraint is given by [13],

$$
I\left(R S_{i}, R S_{j}\right)>I_{\text {inter }} \quad, i, j=1,2, \ldots r, i \neq j
$$

where $I\left(R S_{i}, R S_{j}\right)$ is the distance between two RSs $R S_{i}$ and $R S_{j}$ and $I_{\text {inter }}$ is the interference threshold distance.

2. The placement budget $(P B)$ constraint is given by [10],

$$
\sum_{i=1}^{b} C_{i}^{e N B} \cdot \beta_{i}+\sum_{m=1}^{r} C_{m}^{R S} \cdot \gamma_{m} \leq P B
$$

where $C_{i}^{e N B}$ and $C_{m}^{R S}$ are the cost of the eNB and RS respectively. $\beta_{i}$ and $\gamma_{m}$ are binary variables which are given by, $\beta_{i}=\left\{\begin{array}{l}1 \quad \text { if eNB is placed in } i^{\text {th }} \text { candidate location } \\ 0 \quad ; \text { else }\end{array}\right.$

$\gamma_{m}= \begin{cases}1 & ; \text { if } R S \text { is placed in } m^{\text {th }} \text { candidate location } \\ 0 & ; \text { else }\end{cases}$

3. The coverage ratio constraint is given by

$$
C R \geq E C R
$$

where $C R$ is the obtained coverage ratio and $E C R$ is the expected coverage ratio. The two stage transmission nature of the MHR system may lessen the capacity and leads to transmission delay [12]. The eNB needs to choose for an indirect transmission, exactly when it is needed. This issue is extreme when the UEs are connected by both eNB and RS. Since the objective is to maximize the network throughput and to reduce the delay, the eNB uses TO selection rule to decide about the direct or indirect transmission. The indirect transmission is preferred as long as [16],

$$
R_{e r u}>R_{e u}
$$

where $R_{\text {eru }}$ is the data rate between eNB, RS and UE and $R_{e u}$ is the data rate between eNB and UE.

The data rate of indirect transmission is given by,

$$
R_{e r u}=\frac{P}{t_{e N B-R S-U E}}=\frac{P}{\frac{P}{R_{e r}}+\frac{P}{R_{r u}}}=\left(\frac{1}{R_{e r}}+\frac{1}{R_{r u}}\right)^{-1}
$$

where $t_{e N B-R S-U E}$ is the total two-phase transmission time, $P$ is the packet size, $R_{e r}$ is the data rate between eNB and RS and $R_{r u}$ is the data rate between RS and UE.

Based on the TO scheme, the data rate for a UE is

$$
R=\max \left(R_{e r u}, R_{e u}\right)
$$




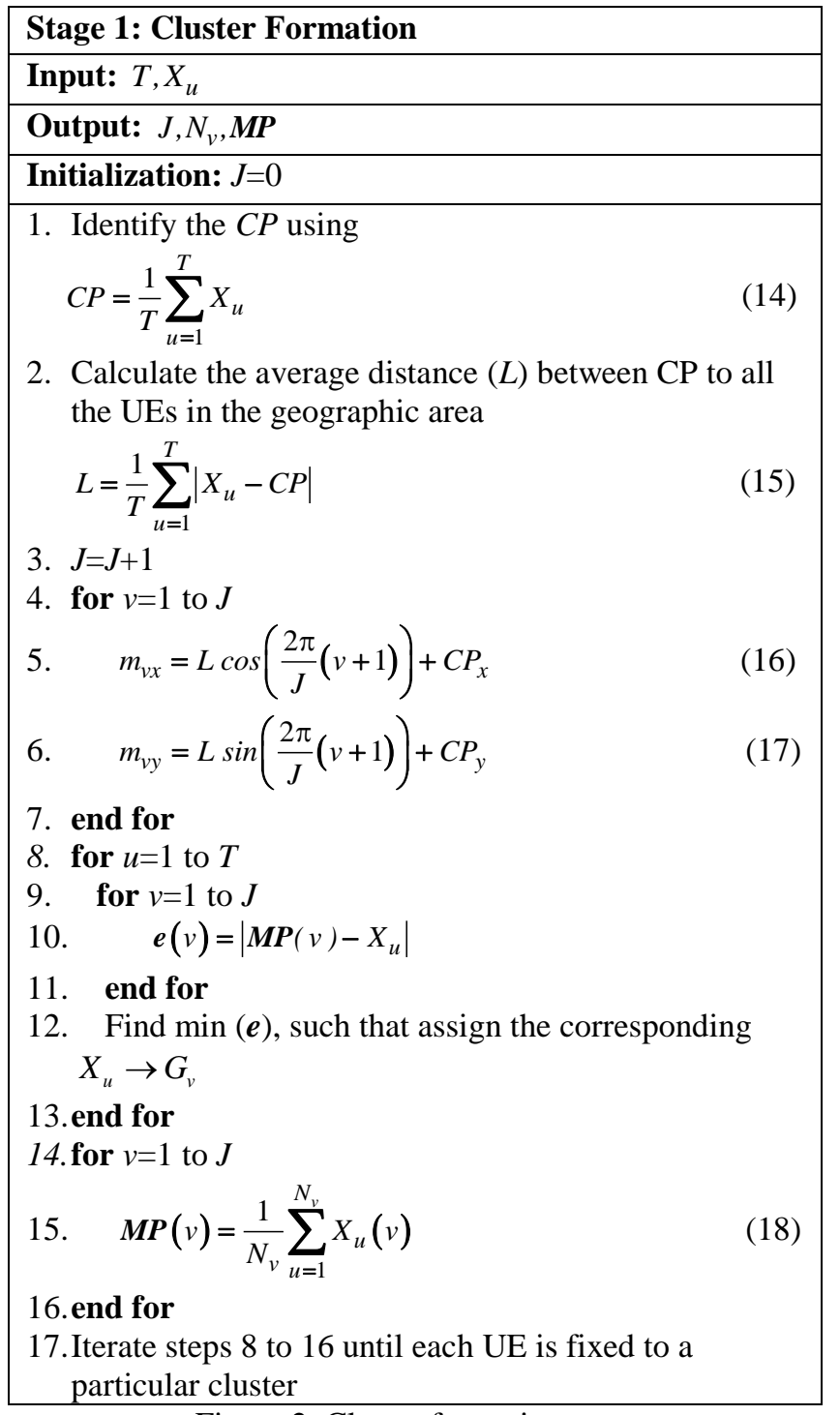

Figure 2. Cluster formation stage

There exists a drawback in the TO scheme. In view of Eq. 13, different UEs may pick the same RS. The functionalities and the capacity of the RS is limited when compared with the eNB. This may prompt a serious issue called network imbalance [14]. This issue may bring about an extreme quality loss in throughput and packet queuing delay. It has been proved that LASER based path determination scheme offers better throughput performance over network imbalance conditions. When the total traffic demand from a specific RS exceeds its capacity, the eNB needs to handover the services of few UEs to the neighbouring RSs from the link overloaded RS. In the event of no neighbouring RS or link overloaded neighbouring RSs, the eNB needs to incline toward for direct transmission.

\section{The proposed eNB and RS placement scheme}

The proposed modified uniform clustering scheme is executed in 2 stages. In the first stage, clusters are formed using uniform clustering algorithm. In the second stage, eNB and RS placement locations are identified with the help of LASER based path determination and TO selection rules. The steps involved in cluster formulation are illustrated in Fig. 2.

The total number of UEs in the given geographic area $(T)$ and the coordinates of UEs $\left(X_{u}\right)$ are given as the input for cluster formation stage. The number of clusters identified $(J)$, number of UEs joined to each cluster $\left(N_{v}\right)$ and the final mean points $(M P)$ are the outputs from this stage. The number of identified clusters is initialized to zero.

Based on the coordinates of UEs, the center point $(C P)$ is calculated in step 1 . The average distance $(L)$ between $\mathrm{CP}$ to all UEs in the geographic area is calculated in step 2. The number of clusters is incremented by one in step 3. The coordinates of the initial MPs are identified from steps 4 to 7 . The distance between every UE to all the MPs are identified from steps 8 to 11 . Each UE is assigned to the cluster $\left(G_{v}\right)$ associated with the nearest MP in step 12. Based on the UEs assigned to every cluster $\left(X_{u}(v)\right)$, the new MPs are identified in step 14 to 16 . These steps are iterated until each UE is fixed to a particular cluster.

The eNB and RS placement stage is illustrated in Fig. 3. In Fig. 3, $B_{i}^{c}$ is the $i^{\text {th }}$ candidate location of eNB, $r^{v}$ is the number of candidate locations of the RSs available in the mentioned cell edge range, $d_{R S}^{k}$ is the average distance from all of the uncovered UEs (UUE) to RS $(k)$ within the coverage range of $\mathrm{RS}(k), \quad \mathrm{T}_{\mathrm{UUE}}$ is the number of UUEs in the geographic region and $D_{R S, k}$ is the total traffic demands of UUE.

For the eNB and RS placement stage, total allocated budget, expected average throughput $\left(R^{E}\right)$, expected coverage ratio, candidate locations of eNB, candidate locations of RS, maximum number of nodes a RS can support $\left(K_{R S}\right)$, interference threshold, number of identified clusters from stage 1 , number of UEs connected to the identified clusters, the identified final MPs and the radius of the eNB $\left(O_{e N B}\right)$ are given as the input for this stage. The total placement cost $(C)$, throughput and coverage obtained, coordinates of the selected eNB $(\boldsymbol{S e N B})$ 


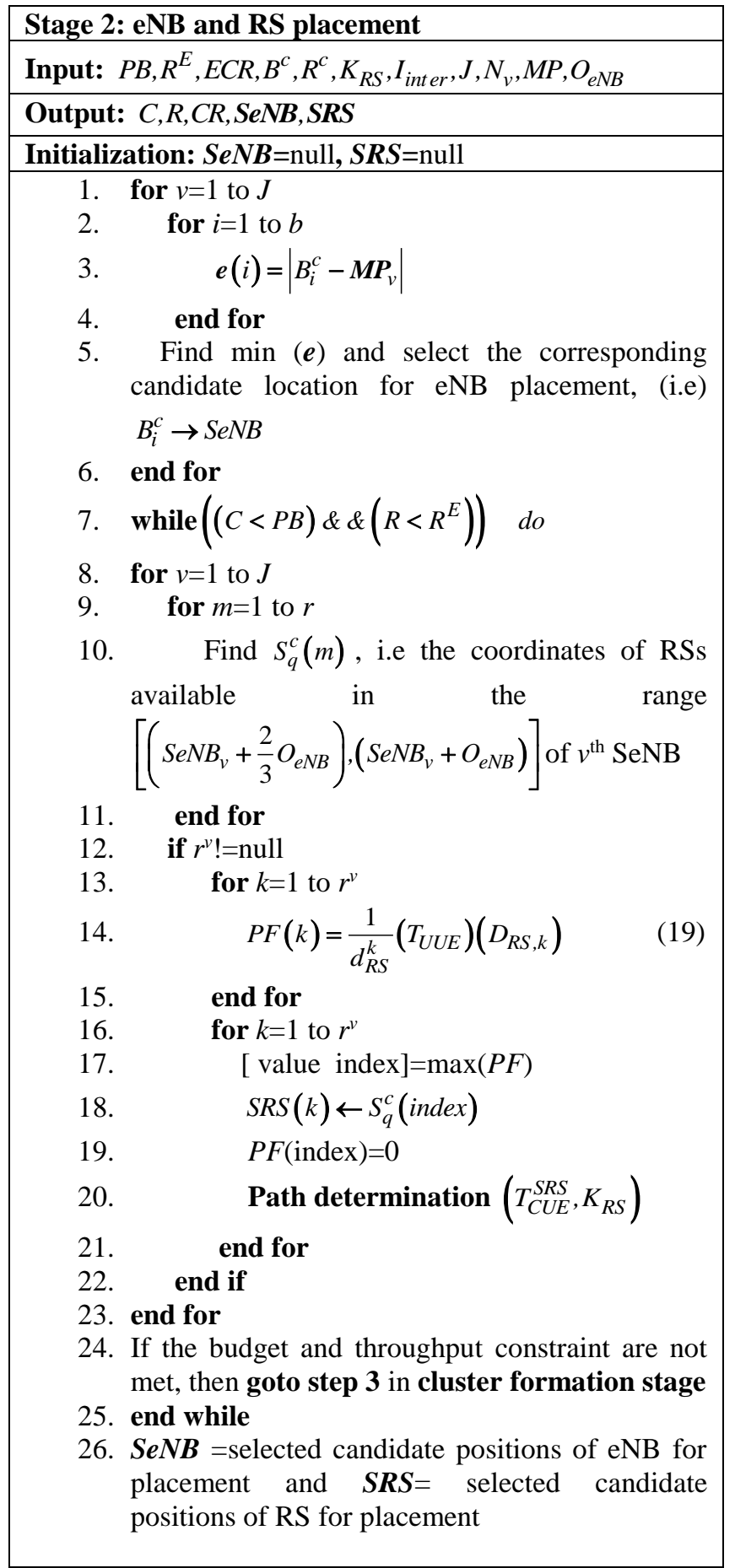

Figure 3. eNB and RS placement stage

and the selected RS (SRS) are the outputs from this stage. The coordinates of $\boldsymbol{S e N B}$ and $\boldsymbol{S R S}$ are initialized to null.

Identify the distance between every final MP to all the candidate locations of eNB. The eNB candidate locations closest to the final MPs are chosen for eNB placement. This is carried out in steps 1 to 6 . To maximize the throughput and coverage, the RS candidate locations at the cell edge are given priority for placement. This also reduces

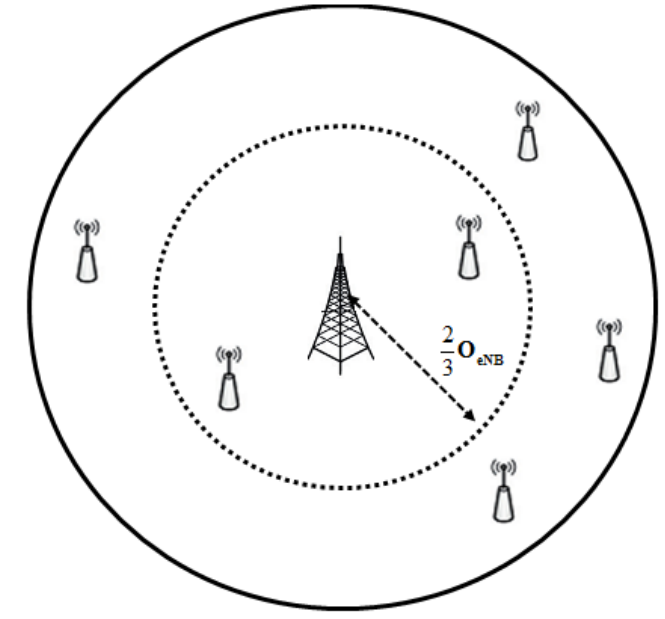

Figure 4. The concept of cell edge RS candidate location selection

the transmission delay and unnecessary path determination. For every selected eNB, identify the candidate locations of RSs in the cell edge (i.e. candidate locations of RSs in the radius range greater than $2 / 3 O_{e N B}$ and lesser than $O_{e N B}$ ). This is carried out in steps 9 to 11. This idea is also illustrated in Fig.4.

If there exist candidate locations of RSs in the above mentioned range, then identify the placement factor $(P F)$ using Eq. (19) for all those RSs. This process is carried out in steps 12 to 15 . Identify the candidate location of RS with highest PF for RS placement.

The corresponding PF index is made to zero so that in the next iteration the candidate location with next maximum PF is chosen. These are done in steps 16 to 19 . In step 20 , the function path determination is called, which minimizes the network imbalance conditions by properly distributing the data traffic between the eNBs and RSs. After the placement of eNBs and RSs, the budget and throughput constraints are verified. If they are not met, then go to step 3 in cluster formation stage. These steps are iterated until the condition in step 24 gets satisfied. Once the budget and throughput requirements are met, the selected eNB and RS locations are stored in $\boldsymbol{S e N B}$ and $\boldsymbol{S R S}$ respectively in step 26 . The procedure for path determination is illustrated in Fig. 5. In Fig. 5, $M_{S R S}$ is the number of SRSs and temp_cap is the capacity of the SRSs after each UE assignment.

The maximum number of nodes a RS can support $\left(K_{R S}\right)$ and the number of covered UEs $(C U E)$ within the range of SRS and outside the range of SeNB $\left(T_{C U E}^{S R S}\right)$ are given as the input for path 


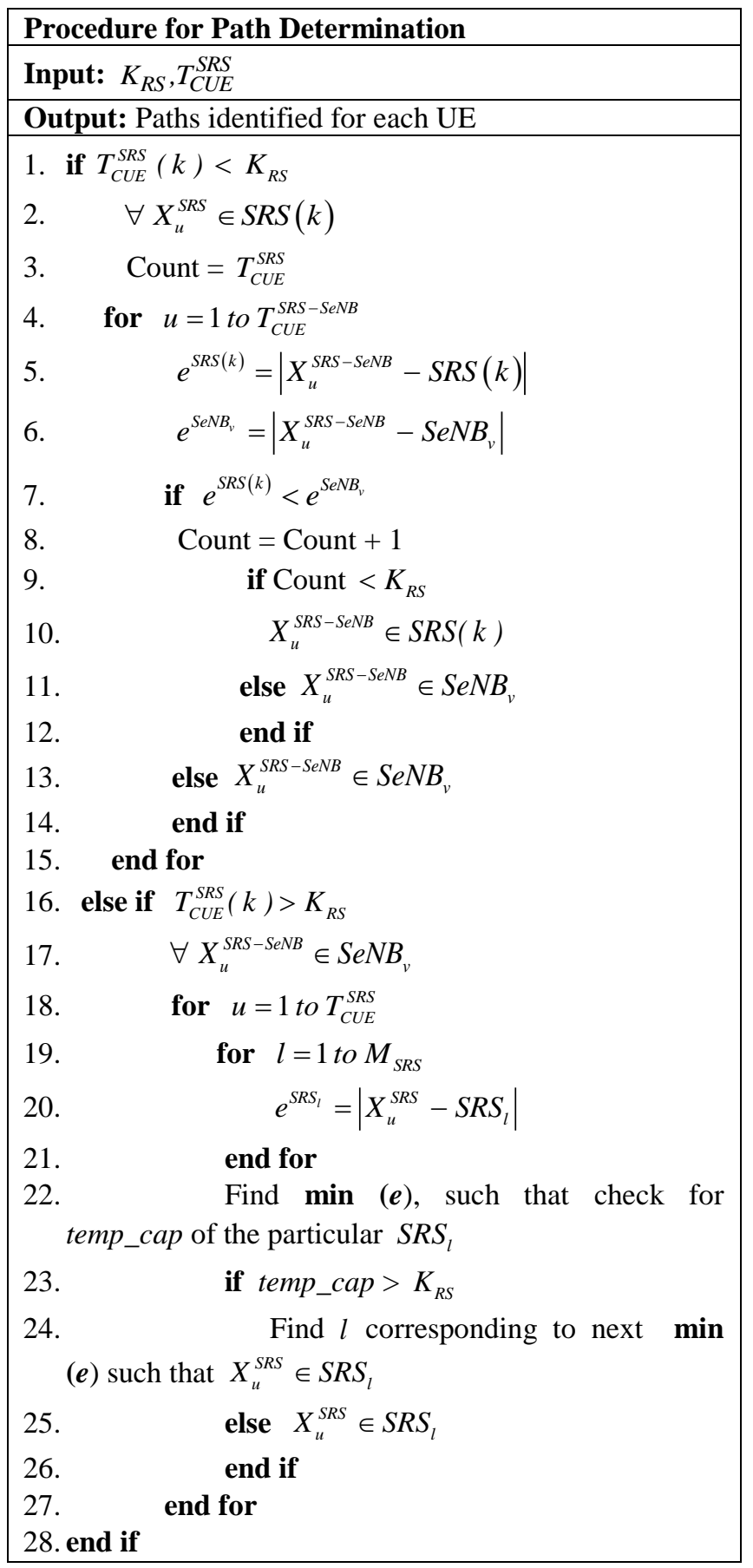

Figure 5. Path determination stage

determination. The paths identified by each UE are the output from this step. If the $T_{C U E}^{S R S}$ of a particular SRS is less than the capacity of RS, then assign all those UEs $\left(X_{u}^{S R S}\right)$ to the corresponding SRS itself.

This is carried out in step 1 and 2 respectively. This idea is illustrated in Fig. 6.

The count is initialized to $T_{C U E}^{S R S}$ in step 3. The following steps are carried out for the UEs which are covered by both SRS and SeNB. The distance

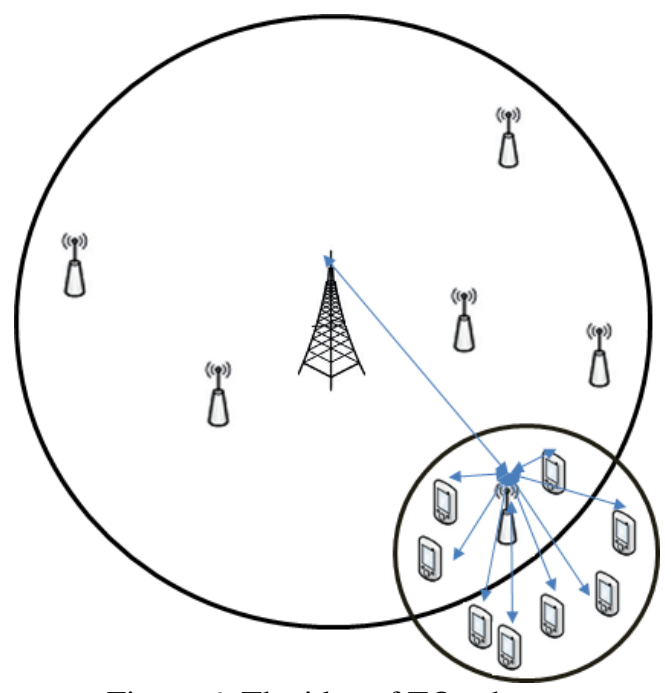

Figure 6. The idea of TO scheme

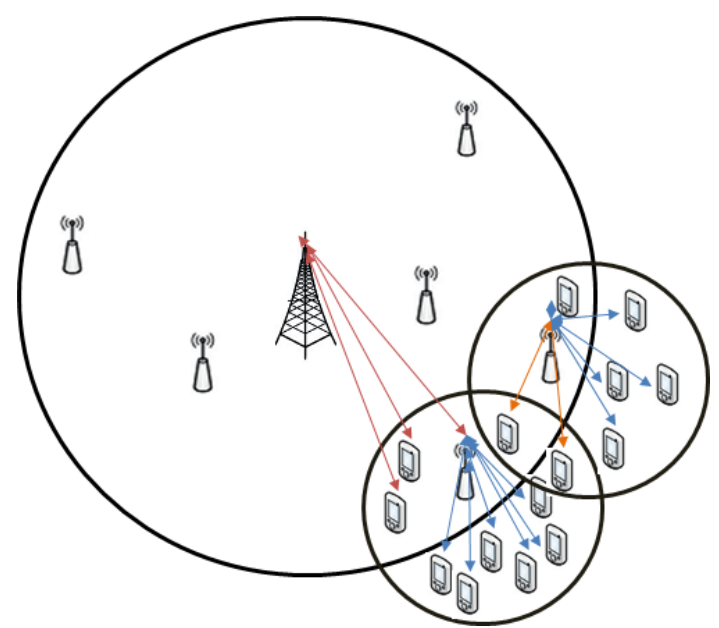

Figure 7. The idea of LASER scheme

between every UE to SeNB and SRS $\left(X_{u}^{\operatorname{SRS}-\operatorname{SeNB}}\right)$ is identified using steps 5 and 6 respectively. If the UE is nearer to SRS, increment count by one. Every time verify the capacity of SRS. If the capacity of that particular SRS is not overloaded, then assign the UE to that SRS. Otherwise assign the UE to SeNB. This is carried out between steps 7 to 15 . This rule is adapted from TO selection scheme.

If the $T_{C U E}^{S R S}$ of a particular SRS is greater than the capacity of RS, then the UEs in the overlapped regions are directly assigned to SeNB based on the availability of the resources. This is done in steps 16 and 17 respectively. Under the network imbalance conditions and no direct connection from SeNB, the UEs in the coverage range of different SRS are identified. Based on the availability of the resources, some of the UEs are handed over to the neighbouring SRS based on the LASER scheme. This is illustrated in Fig. 7. These are done between steps 18 to 28 . The path determination steps are repeated for all the SRSs and SeNBs respectively. 
The path determination rule minimizes the delay and network imbalance.

\section{Simulation results and discussion}

This section of the paper validates the performance of the proposed placement scheme with the simulation results. Y. Chang and Y. S. Lin [10] proved that the uniform clustering based eNB and RS placement scheme offers superior performance than JBRP scheme. Consequently in this work, the performance of the proposed scheme is inspected with the conventional uniform clustering [10], ACRD [18, 19] and JBRP [13] schemes. The simulation study is repeated for 1000 different random UE distributions. The proposed, uniform clustering and JBRP schemes are executed for every UE distribution to find novel eNB and RS placement locations. The average throughput and coverage ratio acquired are taken for the simulation study.

The following parameters are contemplated for our simulation study:

- The square geographic area of size $15 \mathrm{~km} \mathrm{X}$ $15 \mathrm{~km}$ is considered for the eNB and RS placement.

- The candidate locations of the eNBs and RSs are chosen inside the geographic area to be covered.

- The number of candidate locations identified for eNB placement is set to be 6 .

- The candidate locations of RSs are chosen with a minimum separation distance of $0.5 \mathrm{~km}$.

- The number of candidate locations of the RSs is assumed to be $4,8,12,16$, and 20 .

- The UEs are arbitrarily dispersed all through the geographic area.

- UEs are expected to have varying traffic demands in the range of 1 to $10 \mathrm{Mbps}$.

- The coverage range of RS and eNB is set to be 1.9 and $3.2 \mathrm{~km}$ respectively.

- The placement cost of RS and eNB is set to be 3 and 9 units respectively.

- The total placement budget allocated is set to be 50 units.

- The expected average throughput is set to be 8 Mbps.

- The minimum expected coverage ratio is set to be $80 \%$.

A sample simulation scenario is shown in Fig. 8. The number of considered candidate locations of eNB and RS are 6 and 12 respectively. Based on the coordinates of UEs and the number of UEs, the CP is obtained using Eq. (14). The identified CP

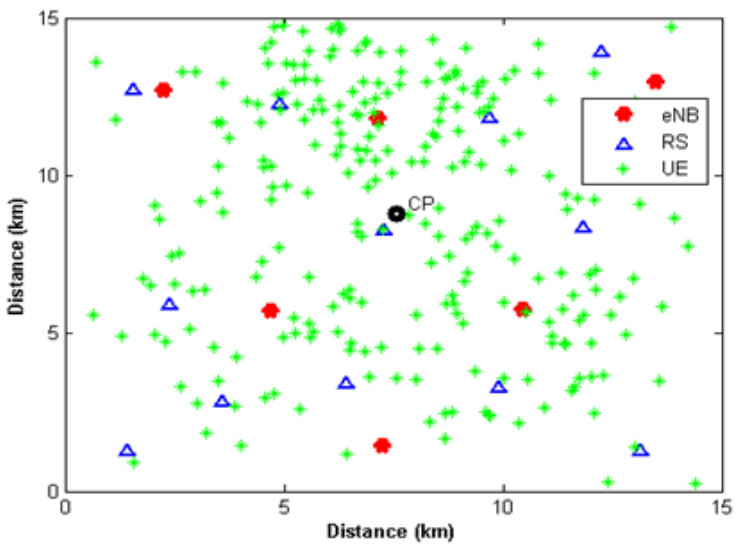

Figure 8. A sample simulation scenario with identified $\mathrm{CP}$

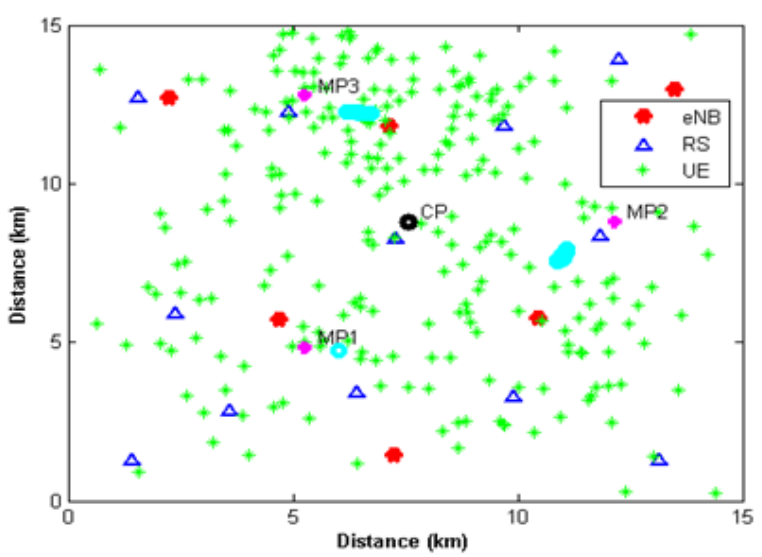

Figure 9. The process of cluster formation

location is marked in Fig. 8. Three clusters are identified to meet the expected throughput, budget and coverage demands. Based on the coordinates of $\mathrm{CP}$, initial MPs are identified using Eqs. (16) and (17). These initial MPs are marked with MP1, MP2 and MP3 in Fig. 9. Then the distance between each UE to every other MP is measured. Each UE will join to its nearest cluster. Based on the UEs associated with every cluster, new MPs are identified using Eq. (18). This process is iterated until each UE is fixed to a particular cluster. The MPs are shifted due to this iteration process, which is also highlighted in the Fig. 9.

The candidate locations of eNBs closer to the identified final MPs are chosen for Macro eNB placement. The 3 SeNBs and the associated coverage range are highlighted in Fig. 10. When the coverage, budget and throughput requirements are not met with the placed Macro eNBs, RSs are introduced. This idea is illustrated in Fig.11. As pointed out earlier, to maximize the coverage and throughput and to minimize unnecessary path determination, RS candidate locations at the cell edge are given higher priority for placement. Based 


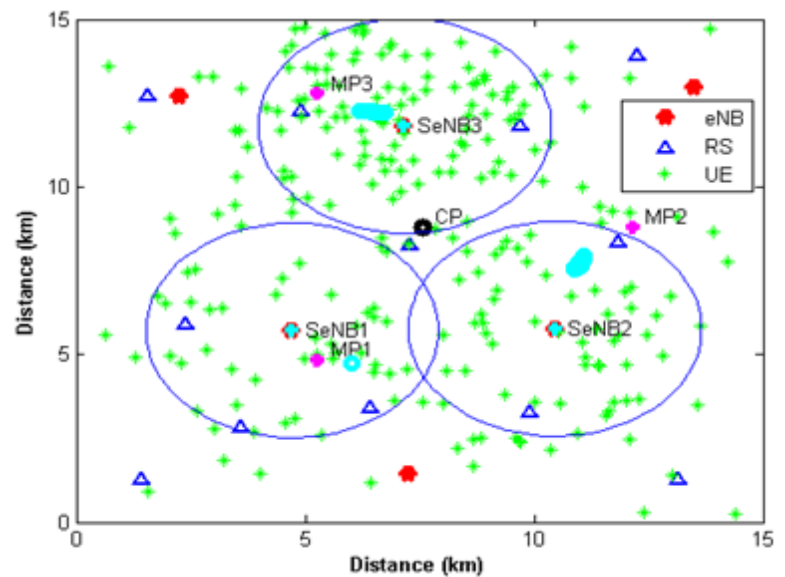

Figure 10. The process of Macro eNB placement

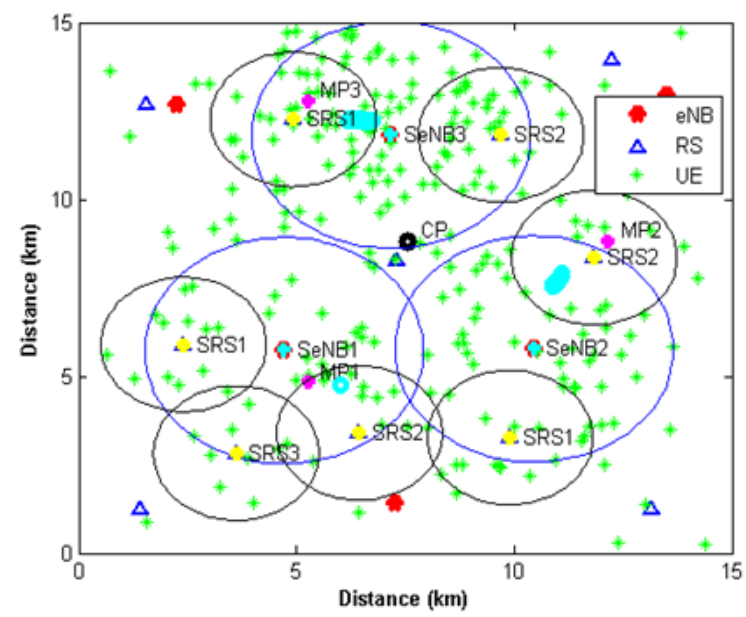

Figure 11. The process of RS placement

on the PF in Eq. (19), 7 candidate locations are identified for RS placement. From Fig.11, it is clear that the identified RS locations are in the cell edge with a separation more than $0.5 \mathrm{~km}$. This also minimizes inter RS interference.

The average per user system throughput for three different deployment schemes is compared in Fig. 12. It is transparent that the increase in the quantity of RS candidate locations will also increase the average system throughput initially. However, after a certain number of RSs, the average throughput remains almost constant because of the issues like inter RS interference. When the number of candidate locations is 12, JBRP [13] scheme offers an average system throughput of $7.78 \mathrm{Mbps}$, which is less than the expected. This is predominantly because of the inappropriate eNB and RS sites. The uniform clustering [10], ACRD [18, 19] and our proposed schemes offer an average system throughput of $8.68 \mathrm{Mbps}, 8.84 \mathrm{Mbps}$ and 8.99 Mbps respectively. Our proposed scheme shows an improvement of $1.67 \%$ over ACRD [18,

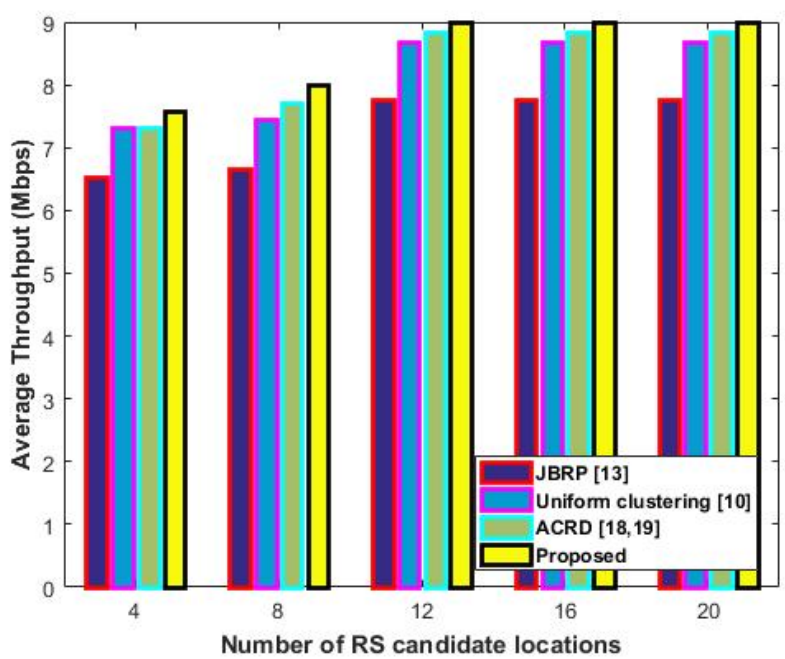

Figure 12. Average system throughput vs. Number of RS candidate locations

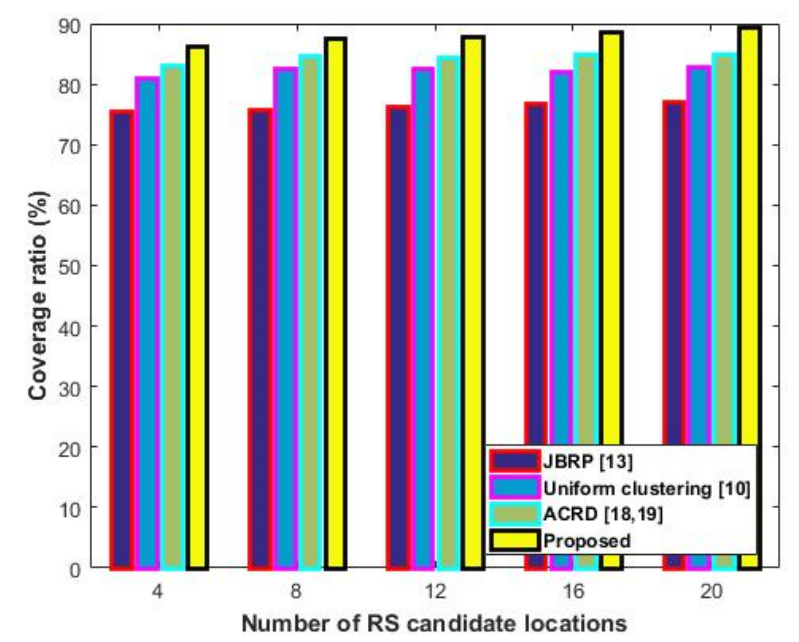

Figure 13. Average coverage ratio (\%) vs. Number of RS candidate locations

19], 3.45\% over uniform clustering [10] and $13.46 \%$ over JBRP [13] schemes. This improvement is due to the robustness of the proposed scheme under network imbalance conditions and also due to the inclusion of LASER and TO selection rules.

The average coverage ratio of three different deployment schemes is compared in Fig. 13. It is transparent that the increase in the number of RS candidate locations is not posing a significant impact on the coverage ratio. The simulation results indicate that the JBRP [13] scheme offers a coverage ratio of $76.46 \%$, when the number of RS candidate locations are 12 , which is less than the expected. The uniform clustering [10], ACRD [18, $19]$ and the proposed schemes offer a coverage ratio of $82.65 \%, 84.56 \%$ and $87.77 \%$ respectively. It is righteous to see that the proposed scheme shows an improvement of $3.21 \%$ over ACRD [18, 19], 5.12\% over the uniform clustering [10] and $11.31 \%$ over the JBRP [13] schemes. It is additionally noticed 
that the average deployment cost of the proposed scheme is 48 units, which is less than the actual allocated deployment budget. Consequently the proposed scheme decreases the cost per bit than the other conventional schemes, which is one of the objectives of this work.

\section{Conclusions}

This work discusses the importance of eNB and RS placement for next generation green cellular networks. Only a very few algorithms addressed the trade-off among the average system throughput, energy efficiency, placement budget and coverage ratio. In this work, we have considered the uniform clustering placement scheme as the base and attempted to enhance its performance by addressing the solutions to its inadequacies. The outcomes acquired demonstrate that the proposed scheme is robust under network imbalance conditions. It is also transparent that the proposed scheme offers improved throughput and coverage ratio under non uniform traffic and network imbalance conditions without claiming additional cost. This is mainly due to the inclusion of TO and LASER schemes and priority for cell edge RS selection. Thus, the proposed scheme is one of the promising candidates for $5 \mathrm{G}$ standard to expand per user throughput and to minimize the energy cost per bit. As a future study, the proposed scheme can likewise be tried with realistic path loss models. Other than RS, the proposed scheme can likewise consider small cells like micro, pico and femto eNBs.

\section{References}

[1] E. Ever, F. M. Al-Turjman, H. Zahmatkesh, and M. Riza, "Modelling green HetNets in dynamic ultra-large-scale applications: A case-study for femtocells in smart-cities", Computer Networks, Vol. 128, pp. 78-93, 2017.

[2] VNI Mobile Forecast Highlights, 2015-2020, 2016, http://www.cisco.com.

[3] M. Arthi and P. Arulmozhivarman, "Fuzzy Logic Based Coverage and Cost Effective Placement of Serving Nodes for $4 \mathrm{G}$ and Beyond Cellular Networks", Wireless Communication and Mobile Computing, Vol. 2017, pp. 1-25, 2017.

[4]M. Arthi and P. Arulmozhivarman, "A Flexible and Cost-Effective Heterogeneous Network Deployment Scheme for Beyond 4G", Arabian Journal for Science and Engineering, Vol. 41, No. 12, pp. 5093-5109, 2016.

[5] Views on Rel-12 and onwards for LTE and UMTS, Future Radio in 3GPP, Huawei Technologies, 2012.
[6] I. F. Akyildiz, D. M. Gutierrez-Estevez, R. Balakrishnan, and E. Chavarria-Reyes, "LTEAdvanced and the evolution to Beyond $4 \mathrm{G}$ (B4G) systems", Phys. Commun., Vol. 10, pp. 31-60, 2014.

[7] M. Arthi, P. Arulmozhivarman, and K. Vinoth Babu, "A quality-aware relay station deployment scheme for green radio communication", Journal of Green Engineering, Vol. 6, No. 2, pp. 1-28, 2016.

[8] M. Arthi, P. Arulmozhivarman, and K. Vinoth Babu, "Quality of service aware multi-hop relay networks for green radio communication", Journal of Green Engineering, Vol. 5, pp. 1-22, 2015.

[9] Y. Yang, H. Hu, J. Xu, and G. Mao, "Relay technologies for WiMAX and LTE-advanced mobile systems", IEEE Communications Magazine, Vol. 47, No. 10, pp. 100-105, 2009.

[10] Y. Chang and Y. S. Lin, "A clustering deployment scheme for base stations and relay stations in multi-hop relay networks", Computers and Electrical Engineering, Vol. 40, No. 2, pp. 407-420, 2014.

[11] J. Cho and Z. J. Haas, "On the throughput enhancement of the downstream channel in cellular radio networks through multi-hop relaying", IEEE Journal on Selected Areas in Communications, Vol. 22, No. 7, pp. 12061219, 2004.

[12] J. H. Huang, L. C. Wang, C. J. Chang, and W. $\mathrm{S} . \mathrm{Su}$, "Design of optimal relay location in twohop cellular systems", Wireless Networks, Vol. 16, No. 8, pp. 2179-2189, 2010.

[13] H. C. Lu and W. Liao, "Joint base station and relay station placement for IEEE $802.16 \mathrm{j}$ networks", In: Proceedings of IEEE global telecommunications conference (GLOBECOM 2009), 2009.

[14] S. S. Wang, C. Y. Lien, W. H. Liao, and K. P. Shih, "LASER, A load-aware spectral-efficient routing metric for path selection in IEEE $802.16 j$ multi-hop relay networks", Computers and Electrical Engineering, Vol. 38, No. 4, pp. 953-962, 2012.

[15] H. K. Chen, H. Yin, J. Liang, Z. N. Li, and S. Y. Liu, "Joint relay station deployment and frequency reuse scheme for two-hop cellular networks", The Journal of China Universities of Posts and Telecommunications, Vol. 19, No.2, pp. 74-80, 2012.

[16] Tutschku, "Demand-based radio network planning of cellular mobile communication systems", In: Proc. of International conference on Seventeenth Annual Joint of IEEE Computer 
and Communications Societies, IEEE INFOCOM '98, Vol. 3, pp. 1054-1061, 1998.

[17] J. Y. Chang and Y. S. Lin, "An Efficient Base Station and Relay Station Placement Scheme for Multi-hop Relay Networks", Wireless Personal Communications, Vol. 82, No. 3, pp. 1907-1929, 2015.

[18] B. J. Chang, Y. H. Liang, and S. S. Su, "Analyses of relay nodes deployment in $4 \mathrm{G}$ wireless mobile multi hop relay networks", Wireless Personal Communications, Vol. 83, No. 2, pp. 11591181, 2015.

[19] C. K. Jishnu, K. Vinoth Babu, M. Arthi, and P. Arulmozhivarman, "A Novel Relay Station Deployment Scheme for beyond 4G Multihop Network", In: Proc. of the International Conference on Emerging Trends in Engineering, Science and Technology (ICETEST), Vol. 24, pp. 864-872, 2015. 